thoroughly as infection with Hepatitis C Virus (HCV). The aim of this audit was to assess the number of service users chronically infected with HBV and their relevant co-infections, co-morbidities and access to treatment.

Methods The Tower Hamlets Specialist Addiction Unit serves the London Borough of Tower Hamlets with a population of more than 200,000. Its Blood Borne Virus Team (BBVT) provides harm reduction healthcare in more than 10 drug and alcohol addiction facilities including outreach sites and neighbouring boroughs. Data on service users chronically infected with HBV was extracted from the service database.

Results Of 2577 people currently registered with the BBVT 49 $(1.9 \%)$ have chronic HBV infection with detectable HBs-Antigen. $88 \%$ are male, the average age is 40 years and the majority is of non-British origin with large groups of Baltic (18\%) and Black/ African/Caribbean (20\%) ethnicity. 16 patients (33\%) are currently injecting drug users (IDU), 13 (27\%) formerly IDU. Other reasons for referral are non-injecting drug and alcohol use. 14/49 patients (29\%) have psychiatric comorbidities other than drug/ alcohol abuse. 22 patients (45\%) knew about their infection when they entered the service.

12 patients (24\%) have HBe-Antigen-positive and 37 (76\%) HBe-Antigen-negative disease. $12 / 49$ patients (24\%) have detectable HCV-RNA and can be regarded as co-infected. $3 / 49$ patients $(6 \%)$ have detectable Hepatitis D Virus (HDV)-RNA. Two patients (4\%) are infected with HBV, HCV and HDV. Two patients (4\%) have active syphilis co-infection and HIV-co-infection, respectively.

$10 / 49$ patients $(20 \%)$ have been diagnosed with cirrhosis. One patient has undergone resection for HBV-associated hepatocellular carcinoma and has been followed up for 9 years without recurrence. Two patients were infected after documented vaccination against HBV. $7 / 49$ patients (14\%) are currently undergoing treatment with a regimen that is effective against $\mathrm{HBV}$. Three patients have cleared HBV, one through treatment and two spontaneously.

Conclusion Even in a difficult setting where care can be interrupted by incarceration or psychiatric deterioration, successful health care for patients with chronic HBV infection is possible by using outreach facilities and appointment reminders. The community attending drug addiction services has overlapping risk factors and in East London, only a minority of patients chronically infected with HBV are currently injecting drug users. Vaccination against HBV has no $100 \%$ protection rate and service users should repeatedly undergo testing for blood borne viruses.

Disclosure of Interest None Declared.

\section{PTH-150 BENEFITS OF A LIVER TRANSPLANT OUTREACH CLINIC: INCREASED REFERRALS AND PATIENT SATISFACTION}

doi:10.1136/gutjnl-2013-304907.637

1,"J L Hulley, ${ }^{1} \mathrm{~A}$ Douglass, ${ }^{2,3}$ S White, ${ }^{2,3}$ S Masson. ${ }^{1}$ Department of Gastroenterology, James Cook University Hospital, Middlesbrough; 'Liver Transplant Unit, Freeman Hos-

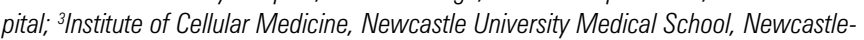
upon-Tyne, UK

Introduction The current landscape of service provision for patients with liver disease does not match that of disease burden ${ }^{1}$. Most hepatologists are based in transplant centres and access to tertiary liver services is not geographically equitable ${ }^{1}$. In an attempt to improve access, we established a liver transplant outreach clinic from the regional liver unit within a large gastroenterology unit. Here, we describe the benefits of this clinic

Methods A dedicated monthly joint liver clinic was established in a large gastroenterology unit. Patients with complex liver disease, including pre- and post-transplant are seen by a consultant transplant hepatologist from the regional liver unit (SM) and a local consultant gastroenterologist (AD). Quantitative data was available from the transplant centre. A sample of patients and specialists were asked to complete a written questionnaire on their opinions of the clinic service.

Results Since August 2010, over 400 patients have been seen. In the 4 years prior to the establishment of the clinic, there were a median of $3(1-4)$ referrals annually for liver transplant assessment. This increased to 9.5 (9-10) in the subsequent 2 years. Patients were satisfied with the clinical service (Table 1) and the majority (95\%) preferred local follow up, citing it as more convenient $(100 \%)$ with easier travel arrangements (100\%). Specialists $(\mathrm{n}=16)$ agreed unanimously that the clinic was more convenient for patients, easy to refer into and improved both accessibility to liver services and communication with the regional liver unit. Most (83\%) felt that it reduced waiting times for specialist opinion.

Abstract PTH-150 Table 1 Patient questions and mean score 1 (low) -5 (high)

\begin{tabular}{lc}
\hline Patient Question & Mean Score \\
\hline Overall quality of care and services & 4.5 \\
Access to specialty care, if needed & 4.4 \\
Skill, experience and training of doctors & 4.6 \\
Respect shown to you by doctors & 4.8 \\
Confidence in the doctor you saw & 4.7 \\
\hline
\end{tabular}

Conclusion Establishing an outreach clinic has increased referrals for transplant assessment. Patients prefer to be seen locally and do not feel this affects their specialist care. They have confidence in the skill and experience of the clinicians they see and rate the quality of care, highly. Referring clinicians are also satisfied with the quality and accessibility of the outreach clinic. Overall, outreach clinics may serve to improve equity of access to transplant services.

Disclosure of Interest None Declared.

\section{REFERENCE}

1. Liver Disease Patient Landscape and Care Provision, 2011, http://www.liver.nhs.uk/ publications/accessed 14th Jan 2013

\section{PTH-151 THE IMPLICATIONS FOR NEW TO FOLLOW-UP RATIOS BASED ON DIAGNOSES ENCOUNTERED IN GASTROENTEROLOGY OUTPATIENT SERVICES}

doi:10.1136/gutjnl-2013-304907.638

1,"K Clark, 'A Bond, ${ }^{1} \mathrm{~A}$ Thuraisingam. ' ${ }^{1}$ Gastroenterology, Wirral University Teaching Hospital, Liverpool, UK

Introduction 'Better Care, Better Values' highlighted the importance of outpatient new to follow up ratios (NFRs) (1). Trusts are encouraged to reduce NFRs or may perform unpaid activity. This has implications for patient care, yet can conflict with Speciality guidelines for follow up. There are no published data on the diagnostic case-mix attending secondary care Gastroenterology appointments nor are any diagnostic data available from Hospital Episode Statistics (HES) for outpatient-based specialties.

Methods We performed a retrospective audit of all Gastroenterology follow-up patients attending Wirral University Teaching Hospital (catchment population 360,000) during a 3-month period. All clinic letters were identified from the hospital IT system and the following information obtained.

- Number of new and follow up attendances per Consultantled clinic

- Primary diagnosis for each follow-up encounter

- Appointment outcome- further follow up or discharge

- Discharge rate per diagnosis

- Number seen in dedicated Specialist Nurse clinics 
Results - 675 new and 1622 follow up appointments were seen by Medical Staff (Consultant, Registrar or SHO) giving an overall NFR of $1: 2.4$

- 516 patients were seen in Nurse-led clinics

- NFR and diagnostic case-mix varied by Consultant team

- Table 1 shows the diagnostic case-mix and outcomes

\section{Abstract PTH-151 Table 1}

\begin{tabular}{lcc}
\hline Primary diagnosis & \% of follow up & \% discharged by diagnosis \\
\hline IBD & 23.5 & 1.6 \\
Chronic liver disease & 15.0 & 2.1 \\
IBS & 5.9 & 39 \\
GORD & 3.4 & 51.85 \\
Coeliac & 3.1 & 6.1 \\
IDA & 2.9 & 30.19 \\
Gallstones/biliary & 2.8 & 13.6 \\
Barrett's & 2.2 & 0.0 \\
Others & 41.2 & 25.5 \\
\hline
\end{tabular}

Conclusion Almost 40\% of secondary care follow up patients are seen with IBD or chronic liver disease. Fewer of these patients are discharged than patients with other diagnoses. In order to improve NFRs we now have primary care discharge pathways for stable patients with coeliac disease and limited colitis. Additional pathways are planned but diagnostic case-mix appears to be a major determinant of NFRs and should be taken into consideration when NFR targets are set.

Disclosure of Interest None Declared.

\section{REFERENCE}

1. NHS Institute for Innovation and Improvement. Converting the potential into reality: 10 steps a provider can take to realise the benefits of Better Care, Better Value indicators.

\section{PTH-152 NURSE DELIVERED DAY CASE PARACENTESIS - A SINGLE CENTRE EXPERIENCE}

doi:10.1136/gutjnl-2013-304907.639

1."L Tobin, ' 10 D Patani, 'S Hood, 'R Sturgess, 'N Stern. 'Gastroenterology, Aintree University Hospital NHS Foundation Trust, Liverpool, UK

Introduction Refractory ascites is a debilitating consequence of end stage liver and other diseases. Treatment options are limited and include recurrent large volume paracentesis (LVP). Admission for LVP requires usage of in-patient beds which are in high demand and have a high cost. Since 2009 we have introduced day case paracentesis and trained a Hepatology clinical nurse specialist (CNS) to perform LVP for stable patients as day cases.

Methods We aimed to evaluate our service development to assess the safety and success rate of day case LVP and particularly a nurse delivered day case LVP service. Initially, day case LVP was coordinated by the CNS with trainees in gastroenterology performing the procedure; subsequently we trained up and assessed the CNS in performing LVP independently. A retrospective audit, evaluating all day case LVP performed since the introduction of the service was performed. Aetiology of ascites and severity of liver disease (Child Pugh), volume drained and complications related to drainage were all recorded. The proportion of cases performed by doctors and the CNS were noted to determine relative outcomes. All cases of LVP for ascites due to cirrhosis were given $20 \%$ human albumin solution as per local protocol.

Results 108 LVP performed (in 42 patients). 62 (57.4\%) performed by the CNS. The cause of ascites was cirrhosis in 36 patients ( 94 LVP) and malignancy in 6 patients (14 LVP). In cirrhotic patients, median Child Pugh score was 8 (range 7-11). 107 (99.1\%) of attempts at LVP were successful with 106 (98.1\%) drains sited with a single needle pass. The volume of ascites drained typically was 12-16 litres (range 3-26). Complications included local skin infection requiring oral antibiotics in 1 case $(0.9 \%)$ and leakage of ascites requiring suturing in $11(10.2 \%)$ procedures. Most cases of local leakage were in those with malignant ascites (63.6\%). There were no long term or serious complications and no unplanned admissions following on from day case LVP. There was no difference in success or complication rate between those LVP performed by the CNS or medical staff.

Conclusion Nurse delivered day case LVP is a safe and effective method of managing patients with refractory ascites. It is a method of relieving the burden on the hospital bed base in a sustainable and safe way. In addition, we would anticipate significant cost savings for this model compared to admission for LVP.

Disclosure of Interest None Declared.

\section{PTH-153 THREE YEAR EXPERIENCE IN A NURSE LED TELEPHONE CLINIC: A RETROSPECTIVE STUDY OF A DISTRICT GENERAL GASTROENTEROLOGY CLINIC.}

doi:10.1136/qutjnl-2013-304907.640

1."M Vinayaga-Pavan, 'V Morgan, 'D Suri. 'Gastroenterology Medicine, Whittington Health, London, UK

Introduction With the demand for patient choice and increasing numbers of outpatients being reviewed in secondary care, a nurse led telephone clinic has proved to be an important part of patients care $^{1}$. Our clinic was first developed in 2009 as a way of providing more efficient follow up care to our patients post procedure.

The Aim of this study was to identify and analyse the current use of our telephone clinic and the type of patients and conditions that are managed by the nursing team.

Methods A retrospective study of all patients enrolled between May 2009 and November 2012 to the telephone clinic (TC) was completed. Demographics, procedure referral reason, attendance and outcome data were analysed. This was compared with our current face to face outpatients (FTFOP) data. Costs of care were estimated using data sourced from NHS tariff 2011-12.

Results There were 1021 individual appointments made of which $807(79 \%)$ appointments were completed, 57 messages were left and 82 patients were unable to be contacted. FTFOP non attendance rate was $28 \%$ vs $20 \%$ for the telephone clinic. $54.3 \%$ of patients were female vs $63.7 \%$ in FTFOP. The majority of patienits, $(85 \%)$ were called with the primary reason of test results. $5.6 \%$ of patients were contacted with the primary objective of review and advice.

Patients problems were seperated where possible into catagories 3.4\% hepatobiliary, 5.8\% indeterminate, 42\% Lower Gastrointestinal (GI), 49\% upper GI. Particular common complaints being addressed included dyspepsia, $19.4 \%$ of total patient referral reasons and $7.4 \%$ change in bowel habit. $77 \%$ of patients were discharged after the telephone consultation with $3.3 \%$ given an open appointment. 9.3\% required specific timed follow up in FTFOP. With current tariffs for non face to face out patient appointments at $£ 55.15$ vs FTFOP of $£ 141.44$ we expect initial annual savings based on an average of 235 consultations a year of $£ 20,278.15$.

Conclusion The telephone clinic has provided a useful adjunct in patient to provider care. The data haves shown that a variety of conditions can be successfully managed and relatively few patients require subsequent follow up in a face to face consultation. The clinic seems to be particularly useful in dealing with clinical symptoms which have algorithmic management, such as dyspepsia. Nonattendance rates were comparable. Patients have anecdotally liked the service for its efficiency and time saving approach. Development of this service will include increased monitoring of patient symptoms as a primary reason for review and integration to the email helpline service established since 2008. 